\title{
US Health Resource Utilization and Cost Burden Associated with Choroideremia
}

\author{
Shaobin Dong' \\ Nicole Tsao ${ }^{2}$ \\ Qiang Hou' \\ Duygu Bozkaya' \\ Bart P Leroy (D) $^{3-5}$ \\ 'Value and Access, Biogen, Cambridge, \\ MA, USA; ${ }^{2}$ Medical Health Outcomes and \\ Research, Biogen, Cambridge, MA, USA; \\ ${ }^{3}$ Department of Ophthalmology \& \\ Center for Medical Genetics, Ghent \\ University Hospital, Ghent, Belgium; \\ ${ }^{4}$ Department of Head \& Skin, Ghent \\ University, Ghent, Belgium; ${ }^{5}$ Division of \\ Ophthalmology \& Center for Cellular \& \\ Molecular Therapeutics, The Children's \\ Hospital of Philadelphia, Philadelphia, \\ PA, USA
}

Purpose: Choroideremia is a progressive, inherited retinal dystrophy that leads to blindness. This study of choroideremia addresses health resource utilization (HRU) and costs from a US payor perspective using insurance claims data. The retrospective analysis used data between January 2013 and December 2018 from the IBM MarketScan Commercial, Medicare Supplemental, and Multi-State Medicaid Databases.

Patients and Methods: Patients having $\geq 1$ claim with an International Classification of Diseases, Ninth or Tenth Edition, diagnostic code for choroideremia (363.55/H31.21) were included; a control group was matched 3:1 to the choroideremia group. Patients were followed for $\geq 6$ months. All-cause HRU and costs were compared between cohorts using generalized linear models adjusted for Charlson Comorbidity Index.

Results: There were 199 and 597 patients in the choroideremia and control groups, respectively; the choroideremia group had a higher mean baseline Charlson Comorbidity Index $(0.47$ vs 0.26$)$. The choroideremia group had a significantly greater mean number of hospital admissions (0.09 vs 0.06), outpatient visits (22.33 vs 11.22), and emergency department visits $(0.41$ vs 0.26$)$ per patient per year than the control group. The choroideremia cohort had higher all-cause total annualized costs than the control cohort $(\$ 15,372$ vs $\$ 9285$ ), primarily driven by outpatient visits (\$8306 vs $\$ 4702$ ). This trend was observed across age categories, particularly among patients aged 20 to 44 years (choroideremia, \$14,544 vs control, \$5953).

Conclusion: The choroideremia group had higher all-cause HRU and total costs versus the control group. These findings provide economic context around HRU associated with choroideremia and help assess the potential impact of novel treatments.

Keywords: vision loss, inherited retinal dystrophy, claims data, health economics

\section{Plain Language Summary}

- In individuals with choroideremia, a rare inherited disease of the retina, vision loss progressively leads to blindness over time.

- Vision loss is associated with substantial economic burdens.

- To better inform US health insurance payors regarding the specific unmet medical needs of people with this condition, this study analyzed US health-care costs from insurance claims from people with choroideremia versus those without choroideremia.

- Specifically, this analysis used 3 different US health insurance claims databases (IBM MarketScan Commercial, Medicare Supplemental, and Multi-State Medicaid Databases) to estimate health resource utilization and costs among people with choroideremia as reported from January 2013 to December 2018.

- A total of 199 people with choroideremia and 597 control individuals were included in this analysis. 
- People with choroideremia had a higher average number of hospital admissions, outpatient visits, and emergency department visits than people without choroideremia (ie, higher health resource utilization).

- People with choroideremia had higher all-cause total costs per year compared with people without choroideremia ( $\$ 15,372$ vs $\$ 9285$, respectively), and this difference was driven by people with choroideremia also having higher outpatient visit costs (\$8306 vs \$4702).

- This is the first claims analysis study in choroideremia addressing health resource utilization and costs from a US payor perspective, providing additional clarity about economic burden for people with this rare disease.

\section{Introduction}

Choroideremia is a rare, $\mathrm{X}$-linked, inherited retinal dystrophy (IRD) resulting in progressive vision loss that ultimately leads to blindness. ${ }^{1-3}$ Symptoms of choroideremia begin as impaired night vision followed by declining peripheral vision, eventually resulting in tunnel vision. ${ }^{3,4}$ Central vision progressively declines in male individuals with choroideremia aged $>40$ years, leading first to severe vision loss and complete blindness in late adulthood. ${ }^{1,4}$ Because of the X-linked nature of the disease, females have either fewer or no symptoms despite obvious fundoscopic signs. 4

Vision loss is associated with substantial functional and emotional burden for patients and caregivers. ${ }^{5-8}$ In a survey of 2044 adults from the general US population, $47 \%$ of respondents reported vision loss as the health outcome with the most debilitating effect on daily living. ${ }^{6}$ Reduced quality of life and loss of independence were reported by $>60 \%$ of these respondents as the most concerning consequences of vision loss. ${ }^{6}$ In a study of 5186 individuals aged $\geq 40$ years from the US populationbased National Health and Nutrition Examination Survey (2005-2008) who underwent frequency doubling technology perimetry testing, severity of visual defects was directly associated with functional disabilities, in particular for activities such as walking and driving that require peripheral vision. ${ }^{5}$ In a Dutch cross-sectional survey of 166 patients with retinitis pigmentosa, vision loss was associated with feelings of frustration, anger, stress, and anxiety. ${ }^{8}$ Furthermore, according to a French survey, $52 \%$ of caregivers of patients with blindness reported effects on their physical and mental welfare. ${ }^{7}$

Global studies show that vision loss incurs substantial economic burdens, including high direct costs associated with health resource utilization (HRU) and indirect costs related to lost productivity, unemployment, and loss of income. ${ }^{9-11}$ An analysis of the socioeconomic impact of IRDs in the United States by a consortium of patient organizations and industry partners estimated costs related to choroideremia between $\$ 484.5$ million and $\$ 1.1$ billion in 2019. ${ }^{12}$ However, no published claims analyses have characterized the economic burden of choroideremia for US payors. The objective of this analysis was to estimate HRU and costs using claims data in US patients with choroideremia. Evaluating the economic impact associated with choroideremia may help better characterize the specific unmet medical needs of patients with this condition and assess the potential impact of novel treatments or services targeting choroideremia.

\section{Materials and Methods}

This retrospective analysis used data from January 2013 to December 2018 in the IBM MarketScan Commercial, Medicare Supplemental, and Multi-State Medicaid Databases (Armonk, NY). Patients with choroideremia were identified as those with $\geq 1$ claim with the International Classification of Diseases, Ninth or Tenth Edition, choroideremia diagnostic code $(363.55 / \mathrm{H} 31.21)$ after a 6-month baseline period without any choroideremia claims. Selection criteria for patients with choroideremia (before matching) are summarized in Supplemental Table 1. A control group of patients without choroideremia was exactly matched $3: 1$ to the choroideremia group by birth year, sex, geographic region, health-care plan type, and drug coverage. All patients were followed for $\geq 6$ months.

Outcomes included all-cause total health-care costs (including costs not related to choroideremia or other ocular conditions), comprising hospital admissions, outpatient physician office visits, emergency department visits, and pharmacy resource use per patient/year. Out-of-pocket patient share of costs included copays, deductibles, and coinsurance.

Characteristics of the study cohorts were summarized with descriptive statistics. Bivariate analyses of all study variables were performed; categorical variables were summarized as the count and percentage of patients in each category, and continuous variables were summarized with the mean (standard deviation [SD]). Health resource utilization and costs were compared using generalized linear regression models and were adjusted by baseline Charlson Comorbidity Index (CCI). Total annualized costs were also examined by sex and by age category ( $\leq 20,20-44,45-64$, and $\geq 65$ years). 
Institutional review board approval was not needed for this retrospective claims analysis.

The claims database was compliant with the Health Insurance Portability and Accountability Act of 1996, and the data used for this study did not include any identifiable patient or investigator information, nor did its analysis involve clinical intervention for or interaction with any patients.

The funder of the study had a role in data analysis and data interpretation. All listed authors, including those employed by the funding source, had the right to approve or disapprove the manuscript, and all authors approved the manuscript.

\section{Results}

There were 199 patients in the choroideremia group and 597 in the control group. Patients in the overall study population had a mean (SD) age of 45.3 (22.4) years, $66 \%$ were male, and $65 \%$ had commercial health insurance (Table 1). The choroideremia group had a higher mean (SD) baseline CCI than the control group (0.47 [0.88] vs 0.26 [0.62]; $p=0.0002$ ).

In the follow-up period after adjusting for CCI, the choroideremia group had significantly higher all-cause HRU versus the control group, including a greater mean (standard error [SE]) number of hospital admissions (0.09 [1.16] vs 0.06 [1.12]; $p=0.0325)$, outpatient visits (22.33 [1.01] vs 11.22 [1.01]; $p<0.0001)$, and emergency department visits $(0.41$ [1.07] vs 0.26 [1.05]; $\mathrm{p}<0.0001)$, per patient per year (Table 2). A larger percentage of patients in the choroideremia group versus the control group filled a prescription in the follow-up period $(82 \%$ vs $74 \%$, respectively), with a numerically higher mean annualized number of prescriptions filled (10.11 vs 9.84; $\mathrm{p}=0.0973$; Table 2).

The choroideremia cohort had significantly higher allcause total health-care costs per patient per year than the control cohort $(\$ 15,372$ vs $\$ 9285 ; p=0.0054)$. Differences in costs were primarily associated with higher mean (SE) annualized outpatient costs for the choroideremia group relative to the control group ( $\$ 8306$ [\$1352] vs $\$ 4720$ [\$777] per patient; $p=0.0221$; Figure 1). A similar distribution of allcause costs was seen in both cohorts, with outpatient costs accounting for a majority of annualized costs per patient in the choroideremia (54\%) and control (51\%) groups, respectively. In analyzing the choroideremia cohort by sex, females were significantly older $(p=0.0021)$ and had a numerically higher mean baseline CCI score than males ( 0.5 vs 0.4 ,
Table I Demographics and Characteristics of the Study Population

\begin{tabular}{|c|c|c|}
\hline Characteristics & $\begin{array}{c}\text { Choroideremia } \\
\quad(n=199)\end{array}$ & $\begin{array}{l}\text { Control } \\
(n=597)\end{array}$ \\
\hline Age, mean (SD), y & $45.3(22.4)$ & $45.3(22.4)$ \\
\hline Male, n (\%) & $|3|(66)$ & $393(66)$ \\
\hline $\begin{array}{l}\text { Region, n (\%) } \\
\text { Northeast } \\
\text { North Central } \\
\text { South } \\
\text { West } \\
\text { Unknown }\end{array}$ & $\begin{array}{l}38(19) \\
40(20) \\
55(28) \\
30(15) \\
36(18)\end{array}$ & $\begin{array}{l}114(19) \\
120(20) \\
165(28) \\
90(15) \\
108(18)\end{array}$ \\
\hline $\begin{array}{l}\text { Payor, n (\%) } \\
\text { Commercial } \\
\text { Medicaid } \\
\text { Medicare } \\
\text { supplemental }\end{array}$ & $\begin{array}{l}|3|(66) \\
36(18) \\
32(16)\end{array}$ & $\begin{array}{c}390(65) \\
108(18) \\
99(17)\end{array}$ \\
\hline $\begin{array}{l}\text { Health plan, n (\%) } \\
\text { PPO } \\
\text { HMO/POS } \\
\text { Other }\end{array}$ & $\begin{array}{l}87(44) \\
36(18) \\
76(38)\end{array}$ & $\begin{array}{l}261(44) \\
108(18) \\
228(38)\end{array}$ \\
\hline $\mathrm{CCl}$, mean $(\mathrm{SD})^{\mathrm{a}}$ & $0.5(0.9)^{*}$ & $0.3(0.6)$ \\
\hline $\begin{array}{l}\mathrm{CCl} \text { group, n (\%) } \\
0 \\
1 \\
2 \\
3 \\
\geq 4\end{array}$ & $\begin{array}{c}|4|(7 \mid)^{*} \\
35(\mid 8) \\
15(8) \\
5(3) \\
3(2)\end{array}$ & $\begin{array}{c}482(81) \\
90(15) \\
17(3) \\
3(1) \\
5(1)\end{array}$ \\
\hline
\end{tabular}

Notes: ${ }^{a} \mathrm{CCl}$ based on data 6 months before the index. ${ }^{*} \mathrm{p}<0.05$.

Abbreviations: $\mathrm{CCl}$, Charlson Comorbidity Index; HMO, health maintenance organization; POS, point of service; PPO, preferred provider organization; SD, standard deviation.

Table 2 Annualized Health Resource Utilization in the FollowUp Period

\begin{tabular}{|l|c|c|c|}
\hline $\begin{array}{l}\text { Per Patient per Year, } \\
\text { LSM (SE) }\end{array}$ & $\begin{array}{c}\text { Choroideremia } \\
\mathbf{( n = 1 9 9 )}\end{array}$ & $\begin{array}{c}\text { Control } \\
\mathbf{( n = 5 9 7 )}\end{array}$ & $\mathbf{p}$ value \\
\hline Inpatient admissions & $0.09(1.16)$ & $0.06(1.12)$ & 0.0325 \\
Outpatient visits & $22.33(1.01)$ & $11.22(1.01)$ & $<0.001$ \\
ED visits & $0.41(1.07)$ & $0.26(1.05)$ & $<0.001$ \\
Pharmacy prescriptions & $10.11(1.01)$ & $9.84(1.01)$ & 0.0973 \\
\hline
\end{tabular}

Abbreviations: ED, emergency department; LSM, least squares mean; SE, standard error.

respectively [p $=0.3769]$; Supplemental Table 2). Although no significant differences in total health-care costs were observed between males and females, females had significantly higher emergency department costs whereas males had significantly higher outpatient visit costs. 


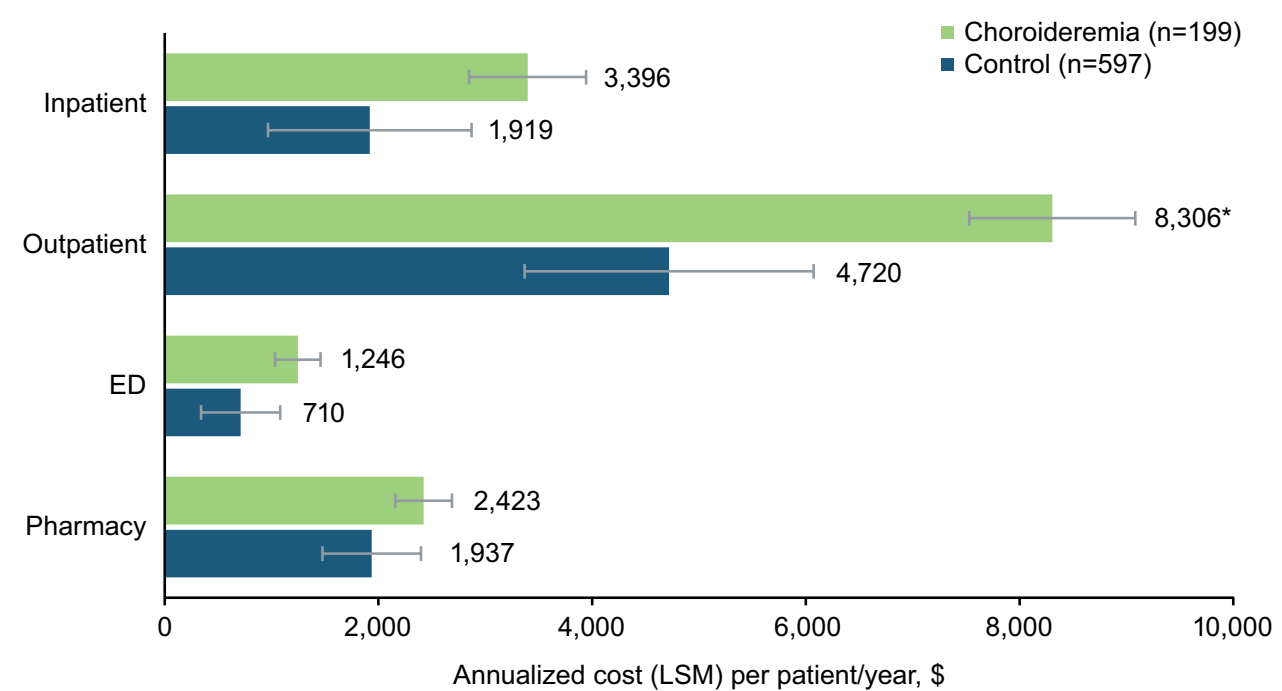

Figure I Annualized costs per patient.

Notes: Error bars are the standard error. ${ }^{*} p=0.0221$.

Abbreviations: ED, emergency department; LSM, least squares mean.

Within the total cost, patient's out-of-pocket costs in the choroideremia group were $\$ 1033$ and $\$ 652$ in the control group (7\% of total costs in both groups; Table 3). Outpatient (choroideremia group, \$707; control group, $\$ 384$ ) and pharmacy prescription (choroideremia group, \$231; control group, \$168) costs accounted for the majority of out-of-pocket patient costs.

When potential drivers of postindex total annualized costs were explored, the percentage of patients with comorbidities accruing costs was higher in the choroideremia cohort across multiple disease categories. Conditions more prevalent in the choroideremia cohort versus the control cohort that were associated with increased costs (Supplemental Figure 1) included (unadjusted odds ratio [95\% CI]) ocular disorders (4.63 [3.30-6.51]), respiratory infection (1.71 [1.22-2.38]), lower respiratory tract disease

Table 3 Patient Share of Annualized Costs ${ }^{\mathrm{a}}$

\begin{tabular}{|l|c|c|}
\hline Per Patient per Year, LSM & $\begin{array}{c}\text { Choroideremia } \\
(\mathbf{n}=199)\end{array}$ & $\begin{array}{c}\text { Control } \\
(\mathbf{n}=597)\end{array}$ \\
\hline Total cost & $\$ 15,372$ & $\$ 9285$ \\
\hline $\begin{array}{l}\text { Out-of-pocket patient share of } \\
\text { annualized costs (\% of total) }\end{array}$ & $\$ 1033(7)$ & $\$ 652(7)$ \\
$\quad$ Inpatient admissions & $\$ 43$ & $\$ 50$ \\
Outpatient visits & $\$ 707$ & $\$ 384$ \\
ED visits & $\$ 52$ & $\$ 49$ \\
Pharmacy prescriptions & $\$ 231$ & $\$ 168$ \\
\hline
\end{tabular}

Note: 'ancludes copays, deductibles, and coinsurance.

Abbreviations: ED, emergency department; LSM, least squares mean.
(1.51 [1.03-2.22]), type 2 diabetes mellitus (1.67 [1.092.56]), and anemia (2.52 [1.34-4.71]).

In an analysis of key subgroups, the choroideremia group consistently had higher mean (SE) annualized allcause costs than the control group across age ranges, particularly among patients aged 20 to 44 years $(\$ 14,544$ [\$2929] vs \$5953 [\$1685]; 144\% difference between groups; $p=0.0119$; Figure 2). No significant differences by sex were observed in mean annualized all-cause costs among patients with choroideremia (males, \$17,669; females, $\$ 18,522 ; p=0.8837$ ).

\section{Discussion}

In this analysis of US claims data, the choroideremia cohort had higher all-cause HRU and total costs compared with the control cohort; these differences were primarily driven by HRU and costs associated with outpatient visits. The choroideremia group had consistently higher all-cause total annualized costs than the control cohort across age groups, with significant differences observed in patients aged 20 to 44 years.

Visual impairment and blindness incur a substantial overall cost burden for patients. Although it can be challenging to directly compare results of health economic analyses due to variations in methodology, patient populations, and global patterns of HRU, findings from this economic analysis of choroideremia were generally equivalent with published estimates of mean annualized costs associated with severe vision loss. A systematic 


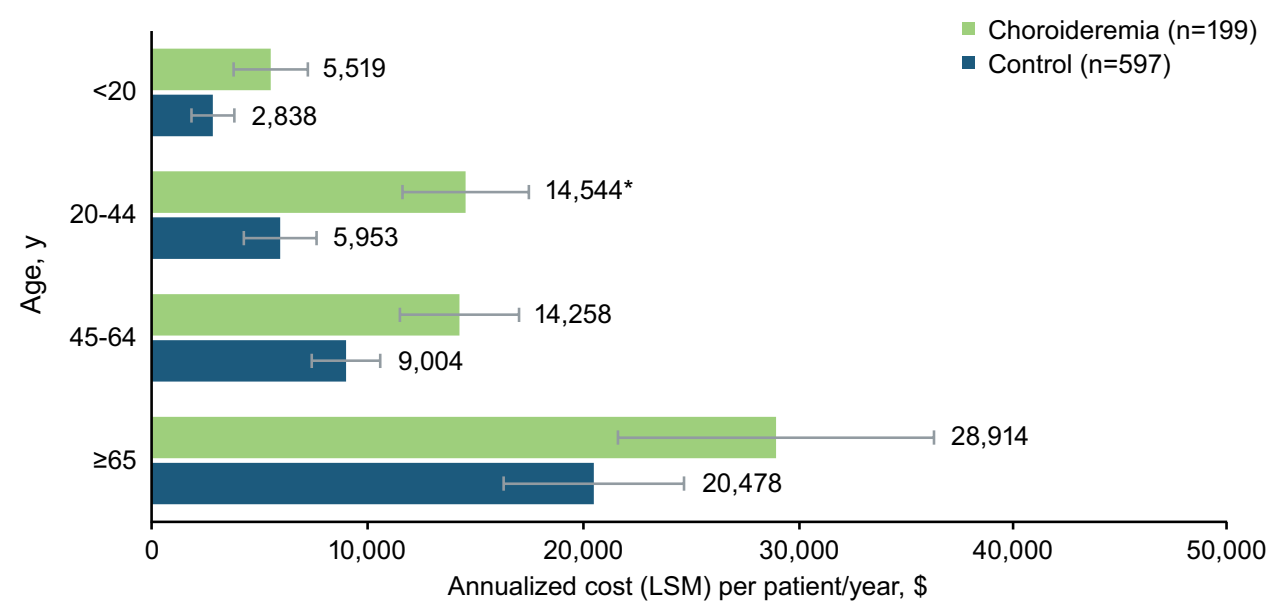

Figure 2 Total annualized costs per patient by age category.

Notes: Error bars are the standard error. ${ }^{*} p=0.0119$.

Abbreviation: LSM, least squares mean.

review of 22 studies related to visual impairment and blindness found mean annualized expenses per person were $\$ 12,175$ to $\$ 14,029$ for moderate visual impairments, $\$ 13,154$ to $\$ 16,321$ for severe visual impairment, and $\$ 14,882$ to $\$ 24,180$ for blindness, showing that cost increases correlated with severity of impairment. ${ }^{10}$ In this analysis, mean annualized costs per patient in the choroideremia group were $\$ 15,372$.

When underlying drivers of costs related to visual impairment were examined in other studies, a breakdown of the costs (millions, \$) of choroideremia in the United States in 2019 showed the greatest share ascribed to loss of wellbeing as measured by years of healthy life lost (301.0-715.8), followed by productivity losses (71.4169.7), caregiver costs (38.5-91.4), health-care system costs (34.4-79.2), loss of taxation revenue (25.5-60.1), and other costs (13.6-32.3). ${ }^{12}$ Other published analyses show a large proportion of the direct costs of vision loss were not associated to eye-related medical care per se, but to falls or accidents due to visual impairment or exacerbation of comorbidities such as diabetes. ${ }^{10}$ Furthermore, a study of patients with IRD demonstrated the substantial impact of indirect costs associated with vision impairment, estimated at $>\$ 1.9$ million over a lifetime, that was primarily attributable to caregiver burden (eg, assistance with activities of daily living) and productivity loss. $^{13}$

In this study, costs of outpatient visits were consistently associated with increased HRU and total costs. The choroideremia group had a higher mean baseline CCI (before first diagnosis in the database), and this could factor into the increased odds observed for several, particularly nonocular, comorbidities that could be a driver of higher costs in this cohort. Additionally, whole metabolomic profiling of 25 individuals with choroideremia found altered lipid metabolism and increased oxidative stress, supporting the possibility of systemic effects of choroideremia, although further research is needed. ${ }^{14}$ In published analyses, visual impairments, such as agerelated macular degeneration (AMD), have also been associated with increased prevalence of chronic comorbidities. For example, cardiovascular and renal conditions were associated with an increased likelihood of early AMD in the US general population. ${ }^{15}$ Studies of Medicare populations demonstrated links between neovascular AMD and incident stroke, particularly among elderly patients ${ }^{16}$ and $>20 \%$ increased odds of hypertension, hypercholesterolemia, emphysema, chronic obstructive pulmonary disease, arthritis, atherosclerosis, and coronary heart disease with neovascular AMD relative to controls. ${ }^{17}$ In a large, retrospective study from Taiwan, incidence of stroke was significantly higher in patients with normal-tension glaucoma versus controls (hazard ratio 6.43; 95\% CI: 4.80-8.38). ${ }^{18}$ Additionally, it is possible that visual impairments could impair ability to engage in regular exercise, indirectly affecting susceptibility to comorbid conditions and potentially health-care costs. Given this pattern of visual impairment and chronic conditions, further investigation is needed to characterize the associations among choroideremia and comorbidities potentially leading to increased outpatient visits and total costs. 
No significant differences in HRU and costs per patient were observed between males and females in this study. This is noteworthy because choroideremia is an X-linked disease, with most female carriers expected to be asymptomatic, ${ }^{19}$ and the present study included a study population composed of $>30 \%$ females. Although this sex difference could be expected to translate into lower costs for females, cost differences were not observed between males and females, potentially because of age-related factors. Females were $\sim 10$ years older than males in this analysis and had $\sim \$ 3000$ higher costs associated with emergency department visits, as well as numerically higher baseline CCI, compared with males. Males, however, had greater outpatient costs than females. Together these data support the possibility that females in this study accrued additional cost burden due to conditions common with advanced age. Another possibility is that costs were influenced by symptom severity. A study of 12 female carriers of disease-causing variants in the CHM gene found that choroideremia symptom severity could manifest as mild, intermediate, or severe ( $\mathrm{n}=4$ in each category). ${ }^{20}$ Because of the nature of the data in the present study, we could not ascertain whether the females included in this analysis were symptomatic or determine the severity of the symptoms, highlighting the need for further studies to assess the percentage of carriers who typically present to the health-care system, to determine whether they are symptomatic, and to investigate differences and similarities in HRU profiles between female carriers and males with choroideremia.

\section{Limitations}

This is the first published study in choroideremia addressing HRU and costs in the United States using claims data; however, there were some limitations to this analysis. The commercial insurance database was primarily employerbased and may not be representative of the overall population. Because patients with more severe visual impairment may be unable to continue employment, ${ }^{10}$ these individuals are likely under-represented in this database. Clinical characteristics, symptoms, and patient age were captured, but severity of choroideremia was not directly assessed. To overcome this limitation, we evaluated outcomes by age categories. Choroideremia is a progressive disease, often leading to blindness in late adulthood, ${ }^{3}$ and age can be considered a reliable proxy measure for disease stage. Because misdiagnosis is common with choroideremia $^{21}$ and genetic testing results were not available to confirm diagnosis, these findings may not be generalizable to the overall population of individuals with choroideremia. Moreover, out-of-pocket costs incurred by patients were evaluated as copayments, but functional adjustments to daily living associated with reduced vision and productivity losses were not assessed (identified as contributing to overall costs of vision loss). Finally, there are limitations inherent to retrospective analyses of claims data that are relevant to the present study, including inability to determine clinical outcomes (eg, extent of visual loss) or implication of potential therapies on cost savings based on claims data. For example, drawing conclusions about costs related to comorbidities in the choroideremia cohort would be supported by laboratory results and clinical assessments, which are unavailable in insurance claims data.

\section{Conclusion}

Choroideremia is a rare disease, and data about the financial burden of this condition are limited. This analysis provides clarity about the economic burden of this condition on males and females. These findings can inform US payors about the excess HRU and cost burden associated with choroideremia and may help to assess the potential impact of novel treatment options for appropriate patient populations with choroideremia.

\section{Acknowledgments}

Writing and editorial assistance were provided by Rebecca Slager, PhD, and Sherri Damlo, ELS, MedThink SciCom, under the direction of the authors with support from Biogen.

\section{Funding}

This study was sponsored by Biogen.

\section{Disclosure}

$\mathrm{SD}, \mathrm{QH}$, and $\mathrm{DB}$ are employees of Biogen and may hold stock in the company. NT is an employee of Sanofi Genzyme and was an employee of Biogen at the time of the study and may hold stock in the company. BPL has received consultancy fees from Bayer, Biogen, GenSight Therapeutics, IVERIC Bio, Novartis Pharma International \& Belgium, Spark Therapeutics Inc, ProQR Therapeutics, REGENXBIO, and Vedere Bio; has received travel support from GenSight Therapeutics, IVERIC Bio, Novartis Pharma International \& Belgium, Spark Therapeutics Inc, and ProQR Therapeutics; has served as a consultant for 
LookoutGTx; and is a senior clinical investigator of the Research Foundation Flanders (grant no. 1803821N). All consultancy fees are paid into accounts that directly support research at BPL's institutions. The authors report no other conflicts of interest in this work.

\section{References}

1. Freund PR, Sergeev YV, MacDonald IM. Analysis of a large choroideremia dataset does not suggest a preference for inclusion of certain genotypes in future trials of gene therapy. Mol Genet Genomic Med. 2016;4:344-358. doi:10.1002/mgg3.208

2. Heon E, Alabduljalil T, McGuigan ID, et al. Visual function and central retinal structure in choroideremia. Invest Ophthalmol Vis Sci. 2016;57:377-387. doi:10.1167/iovs.15-18421

3. Zinkernagel MS, MacLaren RE. Recent advances and future prospects in choroideremia. Clin Ophthalmol. 2015;9:2195-2200. doi:10.2147/OPTH.S65732

4. Pennesi ME, Birch DG, Duncan JL, Bennett J, Girach A. Choroideremia: retinal degeneration with an unmet need. Retina 2019;39:2059-2069. doi:10.1097/IAE.0000000000002553

5. Qiu M, Wang SY, Singh K, Lin SC. Association between visual field defects and quality of life in the United States. Ophthalmology. 2014;121:733-740. doi:10.1016/j.ophtha.2013.09.043

6. Scott AW, Bressler NM, Ffolkes S, Wittenborn JS, Jorkasky J. Public attitudes about eye and vision health. JAMA Ophthalmol. 2016;134:1111-1118. doi:10.1001/jamaophthalmol.2016.2627

7. Brezin AP, Lafuma A, Fagnani F, Mesbah M, Berdeaux G. Prevalence and burden of self-reported blindness, low vision, and visual impairment in the French community: a nationwide survey. Arch Ophthalmol. 2005;123:1117-1124. doi:10.1001/ archopht.123.8.1117

8. Latham K, Baranian M, Timmis M, Pardhan S. Emotional health of people with visual impairment caused by retinitis pigmentosa. PLoS One. 2015;10(12):e0145866. doi:10.1371/journal.pone.0145866

9. Chuvarayan Y, Finger RP, Koberlein-Neu J. Economic burden of blindness and visual impairment in Germany from a societal perspective: a cost-of-illness study. Eur J Health Econ. 2020;21:115-127. doi:10.1007/s10198-019-01115-5

10. Koberlein J, Beifus K, Schaffert C, Finger RP. The economic burden of visual impairment and blindness: a systematic review. BMJ Open. 2013;3:e003471. doi:10.1136/bmjopen-2013-003471

11. Wittenborn JS, Zhang X, Feagan CW, et al. The economic burden of vision loss and eye disorders among the United States population younger than 40 years. Ophthalmology. 2013;120:1728-1735. doi:10.1016/j.ophtha.2013.01.068
12. Deloitte Access Economics. The socioeconomic impact of inherited retinal dystrophies (IRDs) in the United States of America (US); 2020. Available from: https://retina-kyc.org/wp-content/uploads/ 2020/10/DAE-Retina-International-US-Final-

Report_20201008_FINAL.pdf. Accessed December 1, 2020.

13. Jensen IS, Zacherle E, Blanchette C, Kay CN. Estimating the life-time indirect costs of vision impairment in inherited retinal degeneration: economic impact on education, government benefit programs, productivity, and tax loss for patients and caregiver burden. Presented at: American Academy of Ophthalmology Annual Meeting; October 28-30; 2018; Chicago, IL.

14. Lima Cunha D, Richardson R, Tracey-White D, et al. REP1 deficiency causes systemic dysfunction of lipid metabolism and oxidative stress in choroideremia. JCI Insight. 2021;6:e146934. doi:10.1172/ jci.insight. 146934

15. Cheng Q, Saaddine JB, Klein R, Rothenberg R, Chou CF, Il'yasova D. Early age-related macular degeneration with cardiovascular and renal comorbidities: an analysis of the national health and nutrition examination survey, 2005-2008. Ophthalmic Epidemiol. 2017;24:413-419. doi:10.1080/09286586.2017.1337911

16. Liao D, Mo J, Duan Y, et al. Is age-related macular degeneration associated with stroke among elderly Americans? Open Ophthalmol J. 2008;2:37-42. doi:10.2174/1874364100802010037

17. Zlateva GP, Javitt JC, Shah SN, Zhou Z, Murphy JG. Comparison of comorbid conditions between neovascular age-related macular degeneration patients and a control cohort in the medicare population. Retina. 2007;27:1292-1299. doi:10.1097/01. iae.0000300915.81866.b8

18. Lee MS, Kuo LL, Tan EC, Lee OK. Is normal-tension glaucoma a risk factor for stroke? A 10-year follow-up study. PLoS One. 2017;12:e0179307. doi:10.1371/journal.pone.0179307

19. MacDonald IM, Hume S, Chan S, et al. Choroideremia. In: Adam MP, Ardinger HH, Pagon RA, et al. editors. GeneReviews. Seattle: University of Washington; 2020.

20. Jauregui R, Park KS, Tanaka AJ, et al. Spectrum of disease severity and phenotype in chroideremia carriers. Am $J$ Ophthalmol. 2019;207:77-86. doi:10.1016/j.ajo.2019.06.002

21. McLaren TL, De Roach JN, Thompson JA, et al. Expanding the genetic spectrum of choroideremia in an Australian cohort: report of five novel CHM variants. Hum Genome Var. 2020;7:35. doi:10.1038/s41439-020-00122-w
Clinical Ophthalmology

\section{Publish your work in this journal}

Clinical Ophthalmology is an international, peer-reviewed journal covering all subspecialties within ophthalmology. Key topics include: Optometry; Visual science; Pharmacology and drug therapy in eye diseases; Basic Sciences; Primary and Secondary eye care; Patient Safety and Quality of Care Improvements. This journal is indexed on PubMed
Central and CAS, and is the official journal of The Society of Clinical Ophthalmology (SCO). The manuscript management system is completely online and includes a very quick and fair peer-review system, which is all easy to use. Visit http://www.dovepress.com/ testimonials.php to read real quotes from published authors. 